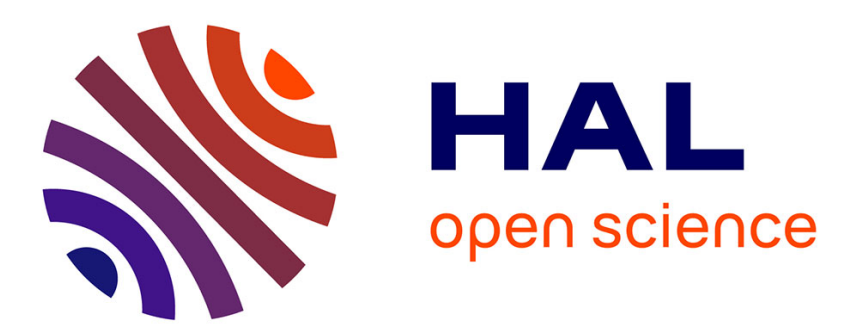

\title{
Local second gradient models and damage mechanics: 1D post-localisation studies in concrete specimens
} Panagiotis Kotronis, F. Collin, René Chambon, Pierre Bésuelle, Jacky Mazars

\section{To cite this version:}

Panagiotis Kotronis, F. Collin, René Chambon, Pierre Bésuelle, Jacky Mazars. Local second gradient models and damage mechanics: 1D post-localisation studies in concrete specimens. Bifurcations, Instabilities, Degradation in Geomechanics, Springer Berlin Heidelberg, pp.127-142, 2007, 978-3-54049342-6. 10.1007/978-3-540-49342-6_6 . hal-01007531

\section{HAL Id: hal-01007531 \\ https://hal.science/hal-01007531}

Submitted on 30 Oct 2019

HAL is a multi-disciplinary open access archive for the deposit and dissemination of scientific research documents, whether they are published or not. The documents may come from teaching and research institutions in France or abroad, or from public or private research centers.
L'archive ouverte pluridisciplinaire HAL, est destinée au dépôt et à la diffusion de documents scientifiques de niveau recherche, publiés ou non, émanant des établissements d'enseignement et de recherche français ou étrangers, des laboratoires publics ou privés. 


\title{
Local Second Gradient Models and Damage Mechanics: 1D Post-Localization Studies in Concrete Specimens
}

\author{
P. Kotronis ${ }^{1}$, F. Collin ${ }^{2}$, P. Bésuelle ${ }^{1}$, R. Chambon1 and J. Mazars ${ }^{1}$ \\ ${ }^{1}$ Laboratoire Sols, Solides, Structures (3S) \\ UJF/INPG/CNRS, \\ Domaine Universitaire, \\ BP 53, 38041 Grenoble cedex 9, \\ France \\ E-mail: Panagiotis.Kotronis@,inpg.fr \\ ${ }^{2}$ Départment Géomac, \\ FNRS-ULG, \\ Liége, \\ Belgium, \\ E-mail: f.collin@ulg.ac.be
}

\section{Introduction}

Continuum damage mechanics is often used as a framework for describing the variations of the elastic properties of due to micro-structural degradations. Experimentally, concrete specimens exhibit a network of microscopic cracks that nucleate sub-parallel to the axis of loading. Due to the presence of heterogeneities in the material (aggregates surrounded by a cement matrix), tensile transverse strains generate a self-equilibrated stress field orthogonal to the loading direction, a pure mode I (extension) is thus considered to describe the behaviour even in compression. This rupture mode must be reproduced numerically. This is the reason why the failure criterion of the chosen constitutive law is expressed in terms of the principal extensions and that a tension test is modelled at the end of this paper. The influence of micro-cracking due to the external loads is introduced via damage variables, ranging from 0 for the undamaged material to 1 for a completely damaged material.

This approach, however, is known to be inadequate for studies where strain softening appears. Calculations performed with a local classical continuum model - which does not incorporate an internal length variable - 
are unable to objectively model intrinsic failure zones. Spurious mesh dependence appears and also cases of failure without any energy dissipation. In order to improve computational performance the nonlocal damage approach is often used in the literature. A different solution is investigated within this work. Local second gradient models are chosen to include a meso scale in the continuous. This approach differs from the nonlocal one in the sense that it is a local theory with higher order stresses depending only on the local kinematic history.

A brief presentation of a classical damage mechanics constitutive law used for the calculations is given at the first part of the paper. The second gradient local approach for a 1D medium is then introduced. Finally, different numerical computations with 1D specimens in traction are presented. Using a random initialization of the iterative solver of the equilibrium equation we search the existence of various solutions and we show that the second gradient term regularize the problem giving results that are mesh insensitive and objective.

\section{Scalar Damage Model}

Introduced in 1958 by Kachanov [1] for creep-related problems, continuum damage mechanics has been applied in the 1980s for simulating the non linear behaviour of concrete [2], [3], [4]. Thermodynamics of irreversible processes gave the framework to formulate the adapted constitutive laws [5], [6]. Considering the material as a system described by a set of variables and a thermodynamic potential, constitutive laws are systematically derived along with conditions on the evolution of damage. However, an adequate choice of the potential and of the damage variable (scalar or tensor) remains to be made. Several anisotropic damage models have already been proposed [7], [8], [9], [10]. Possible applications cover also dynamic problems [11], [12], porous materials [13] and chemical damage [14]. Recent literature reviews on damage mechanics and concrete can be found in [15], [16], [17].

The outlines of a local scalar 3D damage mechanics law for concrete are presented hereafter [18], [19]. In this model, the material is supposed to behave elastically and to remain isotropic. The loading surface takes the following form:

$$
f(D)=\varepsilon_{e q}-K(D)
$$

with $\varepsilon_{e q}$ the equivalent strain defined as: 


$$
\varepsilon_{e q}=\sqrt{\left.\sum_{i=1}^{3}<\varepsilon_{\imath}\right\rangle_{+}^{2}}
$$

being $\varepsilon_{l}$ the principal strains with ( + for traction)

$$
\left.<\varepsilon_{l}\right\rangle_{+}=\varepsilon_{i} \quad \text { if } \quad \varepsilon_{i} \geq 0 \text { and }\left\langle\varepsilon_{l}>_{+}=0 \text { if } \varepsilon_{i}<0\right.
$$

The choice of the previous definition of the equivalent strain $\varepsilon_{e q}$ is guided by the fact that tensile transverse strains are considered to describe the behaviour even in compression (see section 1). $\boldsymbol{D}$ is a scalar whose value is in the domain $[0,1]$. The hardening-softening parameter $\boldsymbol{K}(\boldsymbol{D})$ takes the largest value of the equivalent strain ever reached by the material at the considered point. In that way it retains the previous loading history. Initially $\boldsymbol{K}(0)$ equals a given threshold $\varepsilon_{D 0}$. Evolution laws for damage are used to describe the response in tension or compression - index $\boldsymbol{j}$ refers either to tension $(\boldsymbol{t})$ or compression $(\boldsymbol{c})$ :

$$
D_{j}=1-\frac{\varepsilon_{D 0}\left(1-A_{j}\right)}{\varepsilon_{e q}}-A_{j} \exp \left[-B_{j}\left(\varepsilon_{e q}-\varepsilon_{D 0}\right)\right]
$$

$A_{j}$ and $B_{j}$ are material parameters identified independently from compression tests on cylinders and bending tests on beams. The scalar damage variable $\boldsymbol{D}$, that has to be introduced in the constitutive equation, is a weighted sum of $\boldsymbol{D}_{\boldsymbol{t}}$ and $\boldsymbol{D}_{\boldsymbol{c}}$.

$$
D=\alpha_{t}^{\beta} D_{t}+\alpha_{c}^{\beta} D_{c}
$$

$\beta$ is a parameter that has been added to the original version of the model in order to reproduce more accurately the behaviour of under shear. It is usually taken equal to 1.06 . We call $\sigma_{+}$and $\sigma_{-}\left(\sigma=\sigma_{+}+\sigma_{-}\right)$the tensors in which appear only the positive and negative principal stress respectively, and $\varepsilon_{t}$ and $\varepsilon_{c}$ the strain tensors defined as:

$$
\varepsilon_{t}=\Lambda^{-1}: \sigma_{+} \quad \text { and } \quad \varepsilon_{c}=\Lambda^{-1}: \sigma_{-}
$$

$\Lambda(D)$ is a fourth-order symmetric tensor interpreted as the secant stiffness matrix and it is a function of damage. The weights $\alpha_{t}$ and $\alpha_{c}$ are defined by the following expressions: 


$$
\begin{aligned}
& a_{t}=\sum_{i=1}^{3} H_{i} \frac{\varepsilon_{t i}\left(\varepsilon_{t i}+\varepsilon_{c i}\right)}{\varepsilon_{e q}^{2}} \\
& a_{c}=\sum_{i=1}^{3} H_{i} \frac{\varepsilon_{c i}\left(\varepsilon_{t i}+\varepsilon_{c i}\right)}{\varepsilon_{e q}^{2}}
\end{aligned}
$$

$H_{i}=1$ if $\varepsilon_{i}=\varepsilon_{c i}+\varepsilon_{t i} \geq 0$, otherwise $H_{i}=0 . a_{c}$ and $a_{t}$ are the coefficients defining the contribution of each type of damage for general loading. From (2.7) and (2.8) it can be verified that for uniaxial tension $a_{t}=1, a_{c}=0, D=D_{t}$, and vise versa for compression. Responces under uniaxial compression and tension of this model are presented in figure 2.1 .
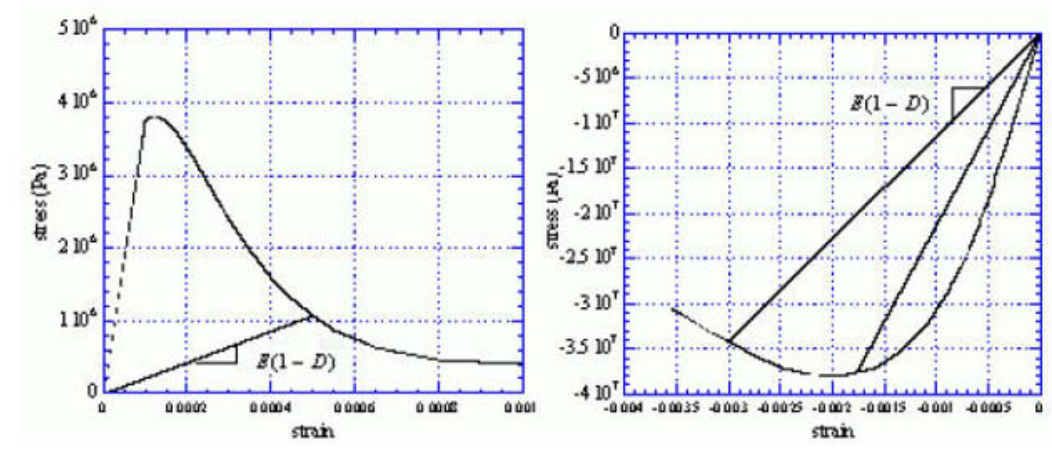

Fig. 2.1. Response of the Mazars damage model for concrete in uniaxial tension (left) and compression (right), (E being the Young modulus)

\section{Local Second Gradient Model}

It is today well established that strain softening induces bifurcation, strain localization, and that the numerical codes often predict failure without any energy dissipation [20],[21]. One of the possible remedies is to use classical damage mechanics constitutive laws based on the nonlocal damage theory (e.g., [22], [23], [24]) or nonlocal second within the flow theory of plasticity. The latter involve the second gradient of the plastic strain in the consistency condition and/or the flow rule, while the kinematic and equilibrium equations remain unchanged (e.g., [25], [26], [27]). For these models the constitutive equation in its incremental form is 
itself a partial differential equation. Peerlings [28], [29] and Fremond [30] have also studied second gradient models within the theory of damage mechanics. Zervos et al. [31] proposed a mixed type model that can be interpreted as a strain-gradient theory with softening law enriched by the second gradient on an internal variable.

A different approach is investigated within this work consisting of using second gradient local models to introduce a meso scale in continuous models [32], [33], [34]. The word local means that the constitutive equation is a relation only between local quantities. Strain gradients are considered as additional observable state variables and are conjugate to higher order stresses that enter the equilibrium equations. This local model is a direct extension of microstructured or micromorphic continua proposed in [35] and [36]. Its general formulation follows.

For a $1 \mathrm{D}$ medium the internal virtual power $P_{\text {in }}^{*}$ for the domain $\Omega=[a, b]$ is a linear form in $\dot{u}^{* \prime}$ and $\dot{u}^{* \prime \prime}$, the first and second derivatives with respect to the space variable $\boldsymbol{x}$ of the virtual velocity field $\dot{u}^{*}($. is the symbol used for the derivatives with respect to the time and ' with respect to the space variable $\boldsymbol{x}$ ). It takes the following form:

$$
P_{i n}^{*}=-\int_{a}^{b}\left(N \dot{u}^{* \prime}+M \dot{u}^{* \prime \prime}\right) d x
$$

$\boldsymbol{N}$ being the usual normal stress in 1D continuum, $\boldsymbol{M}$ is a double stress.

In order to calculate the external virtual power $P_{e x}^{*}$, additional external forces $\boldsymbol{\mu}$, associated to the second gradient terms, have to be added to the classical external forces $\boldsymbol{v}$, associated to the first gradient terms (no distributed forces are taken into consideration, so the only forces are those acting on the two ends $\boldsymbol{a}$ and $\boldsymbol{b}$ ). The virtual power of the external forces is then:

$$
P_{e x}^{*}=v_{b} \dot{u}_{b}^{*}+v_{a} \dot{u}_{a}^{*}+\mu_{b} \dot{u}_{b}^{* \prime}+\mu_{\alpha} \dot{u}_{\alpha}^{* \prime}
$$

The virtual power formulation of the equilibrium equation of the continuum is for all kinematically admissible field $\dot{u}^{*}$ :

$$
P_{e x}^{*}+P_{i n}^{*}=0
$$

Integrating by parts twice shows that equation (3.3) is equivalent to:

$$
N^{\prime}-M^{\prime \prime}=0
$$

and 


$$
\begin{aligned}
& M(a)=-\mu_{\alpha} ; M(b)=\mu_{b} ; \\
& N(a)-M^{\prime}(a)=-v_{a} ; N(b)-M^{\prime}(b)=-v_{b}
\end{aligned}
$$

The next step is to choose the constitutive laws linked with the first and the second gradient part of the model.

$$
\begin{aligned}
& \dot{N}=f\left(\dot{u}^{\prime}\right) \\
& \dot{M}=g\left(\dot{u}^{\prime \prime}\right)
\end{aligned}
$$

For large strain computations, there is a difference between the time derivative of the strains $\varepsilon$ and $\dot{u}^{\prime}$, the derivative of the velocity with respect to the actual space variable. $\varepsilon$ is a Lagrangian measure and $u^{\prime}, \dot{u}^{\prime}$ are Eulerian ones. When small strains are assumed $\varepsilon=u^{\prime}$ and both models can be written in an integral manner as follows:

$$
\begin{aligned}
& N=f(\varepsilon) \\
& M=g\left(\varepsilon^{\prime}\right)
\end{aligned}
$$

The balance equation (3.4) can be integrated as:

$$
N-M^{\prime}=\text { constant }=N_{1}
$$

Substituting $\boldsymbol{N}$ and $\boldsymbol{M}$ given by equations (3.8) and (3.9) in equation (3.10), yields a differential equation which has to be met by the function $\boldsymbol{u}$ of $\boldsymbol{x}$. This equation can be solved analytically under certain conditions. Assuming that there is no coupling between those $\boldsymbol{f}$ and $\boldsymbol{g}$, various types of constitutive relations can be studied. In the original papers [32], [33], a classical elastoplastic bilinear model (or a CLoE family model [37], [38]) exhibiting softening has been chosen for $\boldsymbol{f}$ and a linear relation for $\boldsymbol{g}$. The authors have proven analytically that for a given problem the solutions have to be built using patches of different fundamental solutions, consisted of 'hard parts' corresponding to the unloading pieces and 'soft parts' for the loading pieces of the media. In that way, different solutions are possible (an inner hard solution, a hard-soft solution, a soft-hard-soft solution... see figure 3.1). In order to built a patch solution, one has to equate the values of the displacements $\boldsymbol{u}$, strains $\boldsymbol{u}^{\prime}$ and of the two internal forces $\boldsymbol{N}-\boldsymbol{M}^{\prime}$ and $\boldsymbol{M}$ at the ends of the different pieces in order to meet the virtual power equation, and then to check that $u^{\prime}<e_{\lim }$ in hard pieces and 
$u^{\prime}>e_{\lim }$ in soft pieces ( $e_{\lim }$ being a threshold of the constitutive law, for example $e_{\lim }-\varepsilon_{D 0}$ for the Mazars law).

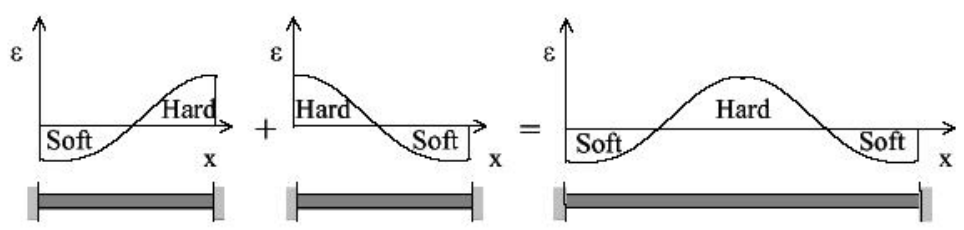

Fig. 3.1. Patches of different fundamental solutions

A 2D second gradient element has been developed [39], [40] and implemented in the finite element code LAGAMINE (Université de Liége). The formulation of the element and the corresponding constitutive equations use a mathematical constraint between the micro kinematics description and the usual macro deformation gradient field. They are valid for large strains. This constraint is enforced in a weak sense by using Lagrange multipliers in order to avoid difficulties with the $\mathrm{C} 1$ continuity (second involving the first and the second derivatives of the displacement field).

In the following section, the feasibility of this local approach with constitutive laws based on damage mechanics is proven numerically using 1D simulations. The Mazars damage mechanics law is used for the function $\boldsymbol{f}$ and some remarks about the uniqueness of solution, the objectivity of the calculations and the influence of a possible coupling between the functions $f$ and $g$ are drawn.

\section{1D Numerical Simulations}

A 1D concrete bar submitted to traction is studied hereafter and the length of the localization zone is compared with the analytical solutions provided in [32], [33]. The bar is modelled using the 2D second gradient element of the finite element code LAGAMINE (Université de Liége, [39], [40]) under plane deformations. In order to avoid any $2 \mathrm{D}$ effects, a zero vertical displacement is applied at the upper and lower boundaries along the bar $\left(\boldsymbol{u}_{2}\right.$ $=0$, figure 4.1). The right end of the bar is fixed $\left(\boldsymbol{u}_{1}=\boldsymbol{u}_{2}=0\right)$ and the external traction displacement is applied at the left end. The additional external forces $\boldsymbol{v}_{\mathrm{b}}, \boldsymbol{v}_{\mathrm{a}}, \boldsymbol{\mu}_{\mathrm{b}}, \boldsymbol{\mu}_{\mathrm{a}}$ are assumed to be zero at both ends. The section of the bar is $0.1 \times 1 \mathrm{~m}^{2}$ and its length $1 \mathrm{~m}$. 


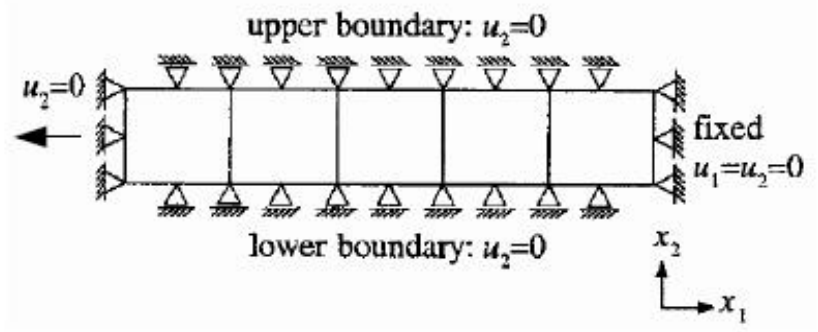

Fig. 4.1. Boundary conditions for simulating $1 \mathrm{D}$ traction in a 2D FE code
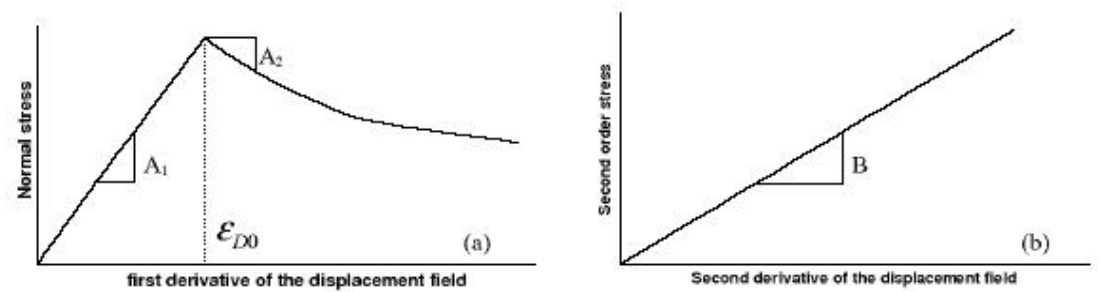

Fig. 4.2. Constitutive models: (a) first gradient part, (b) second gradient part

For the calculations presented hereafter it is assumed that there is no coupling between the first and the second gradient part of the model (functions $\boldsymbol{f}$ and $\boldsymbol{g}$ ). The constitutive relations are the Mazars damage law for the first gradient part and a linear relation for the second gradient part (Figure 4.2). The parameters chosen for the damage law correspond to that of a typical specimen $\quad\left(A_{1}=30 . E+09 P a, \varepsilon_{D 0}=1 . E-04, A_{t}=0.5, B_{t}=2 E+04\right.$, Poisson's ratio 0.2 , parameters that provide $A_{2} \approx-16.7 E+09 P a$ ).

The second gradient local approach defines implicitly two internal lengths, the first (namely $\sqrt{B / A_{1}}$ ) corresponding to the unloading regime of the first gradient part of the model, and the second (namely $\sqrt{B /\left(-A_{2}\right)}$ ) corresponding to the softening loading regime just after the peak ( $\boldsymbol{B}$ being the slope of the linear relation of the second gradient part, see figure 4.2). A way to define the order of magnitude used for $\boldsymbol{B}$, is to use the criteria proposed in [32], [33] - valid under the small strain assumption and for a bilinear plastic law - in order to have possible analytical solutions and to avoid snap-back phenomena for the corresponding differential equation. 
Assuming a two-part solution is possible (built with a patch of a hard part and a soft one) and considering $B=0.37 E+09 N$, one finds analytically that under small strains and for a bilinear law with $A_{1}=30 . E+09 P a$ and $A_{2}=-16.7 E+09 P a$ the length of the soft part is equal approximately to $0.37 \mathrm{~m}$. For the case of a three-part solution (hard - soft - hard) the length of the soft part equals approximately $0.78 \mathrm{~m}$.

As soon as the peak is reached the problem exhibits a loss of uniqueness. In order to determine numerically bifurcation thresholds, an algorithm of random initialization of the iterative solver of the equilibrium equation is used just after the peak (at $\varepsilon_{D 0}=1.2 E-04$, [41], [42]). For every step, a full Newton-Raphson method under displacement control, involving a numerical consistent tangent stiffness operator for the complete model (i.e. the second gradient terms as well as the classical ones) is used [39]. The results of two meshes with 14 and 50 elements are presented hereafter [43]. Figure 4.3 shows the global force displacement curve for both meshes. Figure 4.4 presents the distribution of the damage variable $D$ just after the peak $\left(\varepsilon_{D 0}=1.2 E-04\right)$, and figure 4.5 at the end of the loading $\left(\varepsilon_{D 0}=2.9 E-04\right)$.

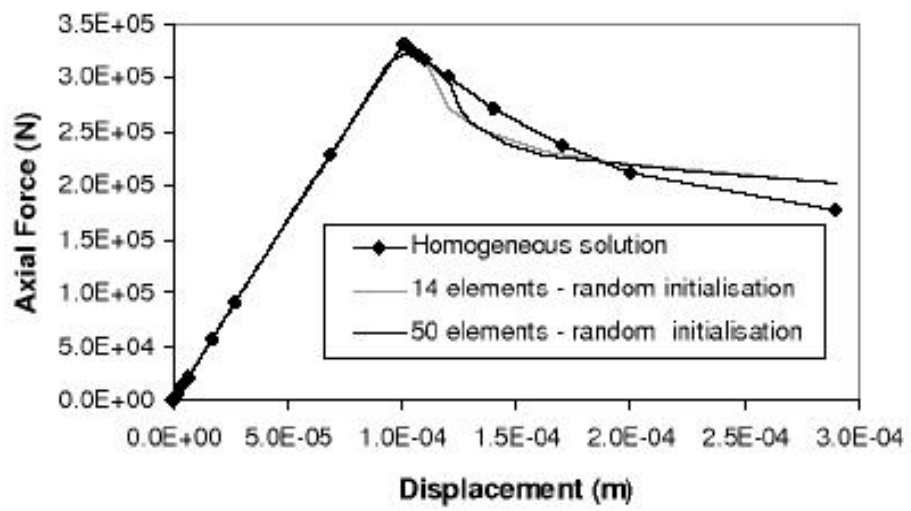

Fig. 4.3. 1D traction: Force - displacement curves for the two meshes 
(a)
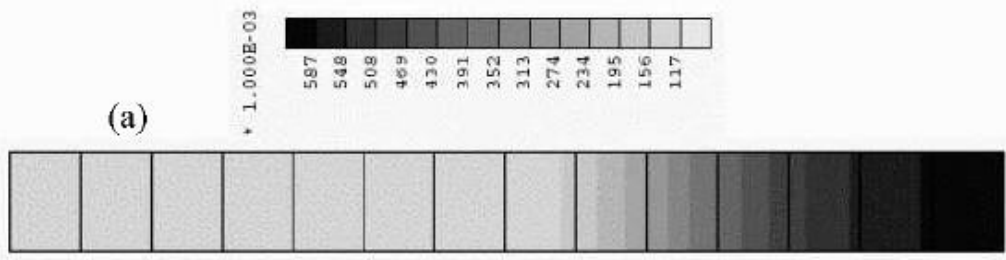

(b)

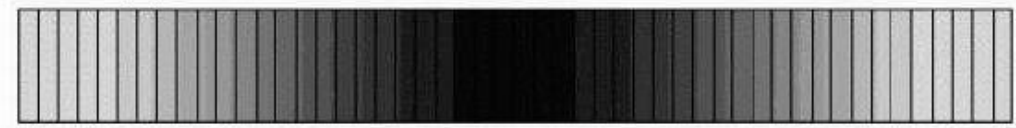

Fig. 4.4. Localization patterns (distribution of damage variable $D$ ) just after the peak $\left(\varepsilon_{D 0}=1.2 E-04\right)$ : (a) 14-element mesh, (b) 50-element mesh

Both meshes reproduce the homogeneous solution when no random initialization of the iterative solver is used. When random initialization of the velocities is used just after the peak, differences in the global curves appear. This is due to the different corresponding localization patterns. The mesh with 14 elements has converged to a solution with two patches (a hard part and a soft one with a length equal approximately to the length calculated analytically). The mesh with 50 elements has converged to a three-part solution (hard - soft - hard) with the length of the soft part again very similar to the analytical value (Figure 4.4). One can also notice that the maximum values of the damage variable $\mathrm{D}$ are different $(0.587$ for the 14 element mesh and 0.347 for the 50 element mesh). Of course, other random initialization can converge to different solutions, independently of the mesh discretization.

The 50-element mesh switches after to the two-part solution (Figure 4.5), thus the localization pattern and the global curves become identical. This is also seen in [44]. The maximum values of the damage variable $\mathrm{D}$ for both meshes are almost the same (approximately equal to 0.876).

From the previous results, it is obvious that the use of local second with damage mechanics laws provide internal lengths, and consequently solutions that do not depend on the spatial discretization. Finite element meshes with different number of elements provide the same solutions 

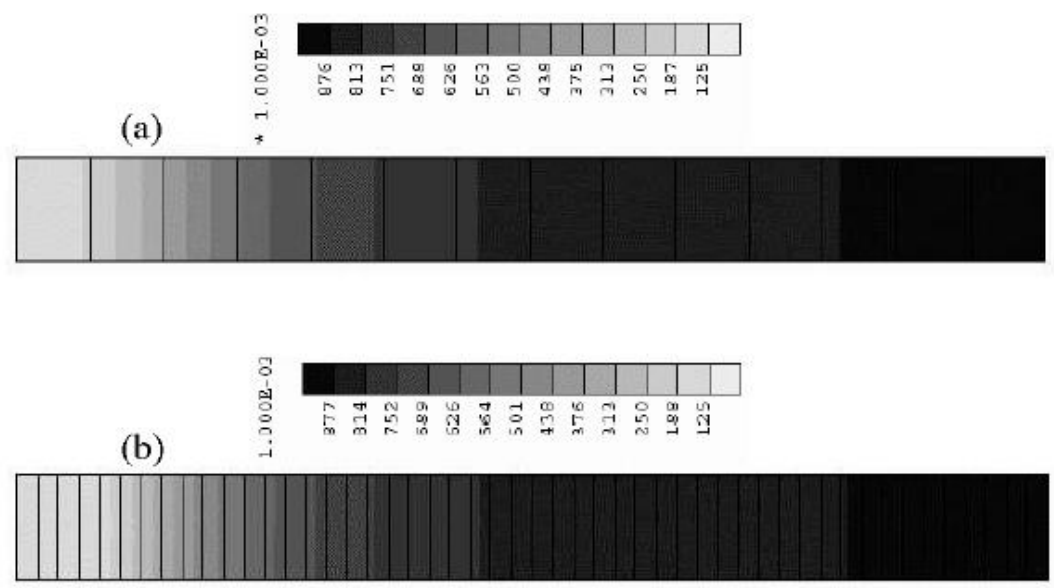

Fig. 4.5. Localization patterns (distribution of damage variable $D$ ) at the end of the loading ( $\varepsilon_{D 0}=2.9 E-04$ ): (a) 14-element mesh, (b) 50-element mesh

(Figures 4.3 and 4.5 at the end of the loading). However, this approach does not restore the uniqueness properties for the corresponding boundary value problem. Different converged solutions can appear (Figures 4.3 and 4.4 just after the peak). Nevertheless, all these solutions are correct and possible to happen, contrary to the parasitic solutions obtained with a classical medium [20], [21].

\section{Expansion of the Plastic Region}

The previous results show that when the magnitude of the softening modulus decreases during the softening process, the plastic region is expanding. Some limited expansion of the plastic region is acceptable. However, as the softening modulus tends to zero, the size of the plastic region can grow without any bounds. This, as mentioned in [45], is a spurious, non physical effect which can be accompanied by stress locking, manifested by a non vanishing residual resistance of the structure even at very large elongations. The total work needed to completely break the bar (given by the area under the load-displacement diagram) is larger than in the absence of localization. In other words, the bar cannot fail by yielding of its limited segments, but every section must sooner or later start yielding. This is in 
contradiction with the observed failure of concrete specimens, typically localized in regions having a limited length.

A way to deal with this problem is to abandon the hypothesis of the non coupling between the first and the second gradient part of the model (functions $\boldsymbol{f}$ and $\boldsymbol{g}$ ). Assuming an anelastic relation also for the function $\mathrm{g}$ (inducing a decrease of the tangent modulus with the loading), one can expect that the structure will not present any residual resistance at very large elongations, and that the spurious stress locking will disappear. The function $\boldsymbol{g}$ could be such that the internal length corresponding to the softening loading regime after the peak stays constant throughout the whole loading history or is a function of the damage variable $D$. In that way we could control the evolution of the localization zone, that can now increase, stay constant or even decrease depending on the form of the chosen function.

For the following simulation the functions $f$ and $g$ are considered coupled. The Mazars damage mechanics law is used for the function $\boldsymbol{f}$ and the material parameters are kept the same as before. However, this time the second order stress $M$ is calculated as a function of the damage variable $D$. Figure 5.1 shows the global force - displacement curves using the second gradient model with or without coupling. Figure 5.2 presents the distribution of the damage variable $D$ during the whole loading when $\boldsymbol{f}$ and $g$ are coupled.

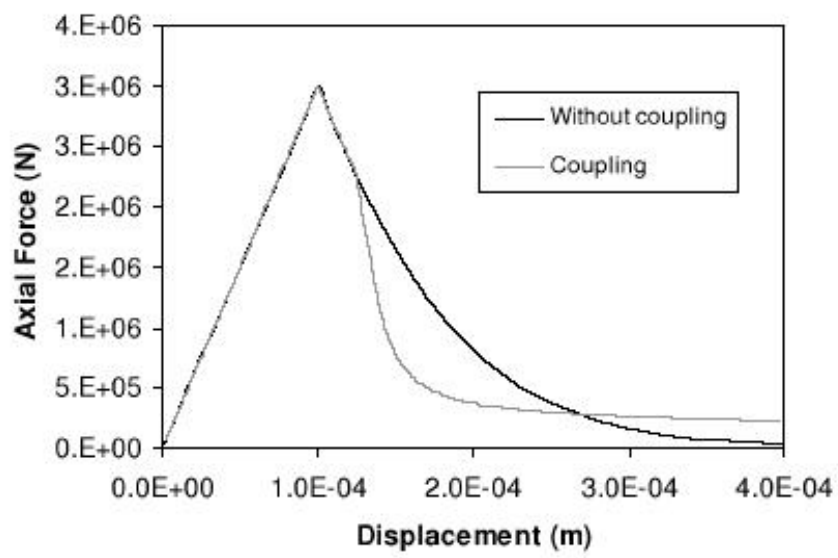

Fig. 5.1. 1D traction: Force - displacement curves considering or not coupling between the first and the second gradient part 
In figure 5.1, one can observe that although the global curve presents a non-zero stress for large elongations, the localization zones in figure 5.2 do not expand. At the beginning and for the first displacement increments the

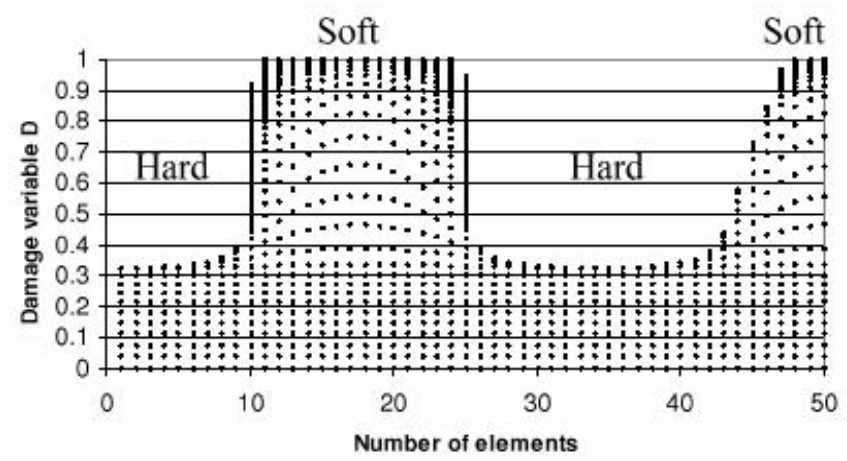

Fig. 5.2. 1D traction: Evolution of the localization pattern for the whole loading history considering coupling between the first and the second gradient part

damage variable has the same value all along the bar. However, a hard soft hard soft solution soon appears and the localization zone does not increase although the damage variable $D$ approaches to 1 . This behaviour is more realistic. The results of this first simulation seem to indicate that the coupling of the first and the second gradient part of the model could provide a solution in order to correctly predict complete failure.

\section{Conclusions}

The feasibility of using local second gradient models with constitutive laws based on damage mechanics is proved throughout this work. 1D numerical computations with concrete specimens and the relevant postlocalization studies are presented. Using a random initialization of the iterative solver of the equilibrium equations it is shown once again that the second gradient term regularizes the problem providing results that are mesh insensitive and objective. However, as expected, it does not restore uniqueness properties for the corresponding boundary value problem. Numerical results considering coupling of the first and the second gradient part of the model indicate that the length of the localization zone can stay constant, property that could be useful in order to reproduce correctly the complete failure of concrete specimens. 
Work in progress concerns the implementation of advanced following path techniques into the finite element code LAGAMINE to reproduce correctly snap-back phenomena [46], and various studies on the boundary conditions to use within a second gradient medium. Numerical simulations of biaxial tests will also be performed and comparisons with experimental 2D failure schemes of various concrete specimens.

\section{References}

1. Kachanov LM (1958) Time of rupture process under creep conditions. Izvestia akademii nauk, USSR, (in Russian), (8):26-31.

2. Krajcinovic D (1983) Constitutive equations for damaging material. Journal of Applied Mechanics ASME, 50:355-360.

3. Lemaitre J, Mazars J (1982) Application de la théorie de l'endommagement au comportement non linéaire et á la rupture du béton de structures. Annales de l'ITBTP, 4010.

4. Ladevéze P (1983) Sur une théorie de l'endommagement anisotrope. Report n. 34, Laboratoire de Mécanique et Technologie, Cachan, France.

5. Lemaitre J, Chaboche JL (1985) Mécanique des matériaux solides. DunodBordas Ed. Paris, France.

6. Lemaitre J, Chaboche JL (1994) Mechanics of Materials. Cambridge University Press, Cambridge, pp. 556.

7. Dragon A, Mroz Z (1979) A continuum model for plastic-brittle behaviour of rock and concrete. International Journal of Engineering Science, 17:121137.

8. Fichant S, Pijaudier-Cabot G, La Borderie C (1997) Continuum damage modelling: approximation of crack induced anisotropy. Mech. Res. Comm., 24:109-114.

9. Fichant S, La Borderie C, Pijaudier-Cabot G (1999) Isotropic and anisotropic descriptions of damage in concrete structures. Mechanics Cohesive Frictional Materials, 4:339-359.

10. Mazars J, Pijaudier-Cabot G (1989) Continuum damage theory - application to concrete. Journal of Engineering Mechanics ASCE, 115:345-365.

11. La Borderie CL (1991) Phénoménes unilatéraux dans un matériau endommageable: modélisation et application ‘a l'analyse des structures en béton. Thése de doctorat, Université Paris 6.

12. Ragueneau F, La Borderie C, Mazars J (2000) Damage Model for Concrete Like Materials Coupling Cracking and Friction, Contribution towards Struc-tural Damping: First Uniaxial Application. Mechanics Cohesive Frictional Materials, 5:607-625.

13. Pijaudier-Cabot G, Burlion N (1996) Damage and localisation in elastic materials with voids. Mechanics Cohesive Frictional Materials, 1:129-144. 
14. Gérard B, Pijaudier-Cabot G, La Borderie C (1998) Coupled DiffusionDamage Modelling and the Implications on Failure due to Strain Localisation. International Journal of Solids Structures, 35:4107-4120.

15. Pijaudier-Cabot G (2000) Continuum damage modelling. Revue Française de Génie Civil, 4(5):33-57. Numéro spécial, Ecole d'été ALERT, Constitutive Modelling of Geomaterials. Cambou, Di Prisco (eds).

16. Pijaudier-Cabot G, Mazars J (2001) Damage models for concrete. In Handbook of Materials Behaviour Models. Lemaitre J (ed.) Chapter 6Damage models. Academic Press: New York, 500-513.

17. Jirasek M, Bažant ZP (2001) Inelastic Analysis of Structures. John Wiley and Sons (eds), 758 pages, Chichester, November.

18. Mazars J (1984) Application de la mécanique de l'endommagement au comportement non linéaire et á la rupture du béton de structure. Thése de Doctorat d'Etat dés Sciences Physiques, Université Pierre et Marie Curie Paris 6, France.

19. Mazars J (1986) A description of micro and macroscale damage of concrete structures. Engineering Fracture Mechanics, 25(5/6):729-737.

20. Bažant ZP (1976) Instability, ductility and size effect in strain softening concrete. Journal of Engineering Mechanics ASCE, 102:331-344.

21. Bažant ZP (1984) Continuum theory for strain softening Journal of Engineering Mechanics ASCE, 110:1666-1692.

21. Pijaudier-Cabot G, Bažant ZP (1987) Nonlocal damage theory. Journal of Engineering Mechanics ASCE, 113:1512-1533.

22. Pijaudier-Cabot G, Haidar K, Dubé JF (2004) Non-local damage model with evolving internal length. International Journal for Numerical and Analytical Methods in Geomechanics, 28:633-652.

23. Jirásek M (2004) Non-local damage mechanics with application to concrete. Revue française de Génie Civil, 8:683-707.

24. Aifantis E (1984) On the mirostructural origin if certain inelastic models. Transaction of ASME, J. Mat. Engng. Tech. 106:326-330.

25. Vardoulakis I, Aifantis E (1991) A gradient flow theory of plasticity for granular materials. Acta Mech., 87:197-217.

26. De Borst R, Muhlhaus H (1992) Gradient dependent plasticity: formulation and algorithmic aspects. International Journal of Numerical Methods in Engineering, 35:521-539.

27. Peerlings RHJ, de Borst R, Brekelmans WAM, de Vree JHP (1996) Gradient enhanced damage for quasi-brittle materials. International Journal of Numerical Methods in Engineering, 39:3391-3403.

28. Peerlings RHJ, Geers MGD, de Borst R, Brekelmans WAM (2001) A critical comparison of nonlocal and gradient-enhanced softening continua. International Journal of Solids Structures, 38:7723-7746.

29. Fremond M, Nedjar B (1996) Damage gradient of damage and principle of virtual work. International Journal of Solids Structures, 33(8):1083-1103.

30. Zervos A, Papanastasiou P, Vardoulakis I (2001) A finite element displacement formulation for gradient elastoplasticity. International Journal of Numerical Methods in Engineering, 50:1369-1388. 
31. Chambon R, Caillerie D, El Hassan N (1996) Etude de la localisation unidimensionnelle á l'aide d'un modéle de second gradient. C.R.A.S-Série IIb, 323:231-238.

32. Chambon R, Caillerie D, El Hassan N (1998) One dimensional localisation studied with a second grade model. European Journal of Mechanics A/Solids, 17(4):637-656.

33. Chambon R, Caillerie D, Matsushima T (2001) Plastic continuum with microstructure, local second gradient theories for geomaterials: localization studies. International Journal of Solids and Structures, 38:8503-8527.

34. Mindlin RD (1964) Micro-structure in linear elasticity. Arch. Ration. Mech. Anal, 16:51-78.

35. Germain P (1973) The method of virtual power in continuum mechanics. Part 2 : Microstructure. SIAM J. Appl. Math., 25:556-575.

36. Chambon R (1989) Une classe de lois de comportement incrémentalement non linéaires pour les sols, résolution de quelques problémes de cohérence. C.R.A.S. Série II., 308:1571-1576.

37. Chambon R, Desrues J, Charlier R, Hammad W (1994) CLoE, A new rate type constitutive model for geomaterials: Theoretical basis and implementation. International Journal for Numerical and Analytical Methods in Geomechanics, 18(4):253-278.

38. Matsushima T, Chambon R, Caillerie D (2000) Second gradient models as a particular case of microstructured models: a large strain finite element analysis. C.R.A.S-Série II b, 328:179-186.

39. Bésuelle P (2003) Implémentation d'un nouveau type d'élément fini dans le code LAGAMINE pour une classe de lois á longueur interne. Rapport d'activité pour le FNRS, Belgique, mai.

40. Chambon R, Crochepeyre S, Charlier R (2001) An algorithm and a method to search bifurcation points in nonlinear problems. International Journal of Numerical Methods in Engineering, 51:315-332.

41. Chambon R, Moullet JC (2004) Uniqueness studies in boundary value problems involving some second gradient models. Computer methods in applied mechanics and engineering, 193:2771-2796.

42. Kotronis P, Chambon R, Mazars J, Collin F (2005) Local second gradient models and damage mechanics: Application to concrete. 11th International Conference on Fracture, Turin, Italy, Org. ICF, cd paper no 5712, 20-25 March.

43. Bésuelle P, Chambon R, Collin F (2006) Switching mode of deformation in post-localization solutions with quasi brittle material. Journal of mechanics of materials and structures in print.

44. Rolshoven S (2003) Nonlocal plasticity models for localised fracture. Thése N0 2887, Ecole Polytechnique Fédérale de Lausanne.

45. Kotronis $P$, Collin F (2005) Implementation of following path techniques into the finite element code LAGAMINE. Rapport Interne G'eomac/3S, septembre. 